\title{
Capacity Analysis for MIMO Two-Hop Amplify-and-Forward Relaying Systems with the Source to Destination Link
}

\author{
Abdulla Firag $\ddagger \quad$ Peter J. Smith ${ }^{\ddagger} \quad$ Matthew R. McKay ${ }^{\dagger}$ \\ ${ }^{\ddagger}$ Dept. of Electrical and Computer Engineering, University of Canterbury, Christchurch, New Zealand \\ ${ }^{\dagger}$ Dept. of Electronic and Computer Engineering, Hong Kong University of Science and Technology, Hong Kong
}

\begin{abstract}
This paper presents an ergodic capacity analysis of an amplify-and-forward multiple-input, multiple-output two-hop system including the source to destination (direct) link. We first derive an expression for the probability density function of an unordered eigenvalue of the system. Then, using this result, a closed form expression for the ergodic capacity of the system is derived. The ergodic capacity expression has one integral that needs to be evaluated numerically. The results produced are valid for all SNR values and for arbitrary numbers of antennas at the source, relay and destination. We also present simulation results to validate our analysis. The results show that the analysis exactly matches the simulations and quantifies the improvements in capacity due to the diversity offered by the direct link.
\end{abstract}

\section{INTRODUCTION}

Wireless relaying networks have recently been given considerable attention due to their many advantages. Apart from increasing the range, relaying networks can also achieve better diversity by using cooperative transmission from the source and several relays [1], [2]. The relaying terminals forward the information from the source to the destination mainly using two well known methods: amplify-and-forward (AF) and decode-and-forward (DF). In AF mode, the relay terminal does not decode or demodulate, but amplifies the received observations, corresponding to the signal from the source, and retransmits to the destination. Since multiple-input, multipleoutput (MIMO) systems can provide better system capacity than single-input, single-output (SISO) systems, relaying has recently been extended to MIMO scenarios [3], [4]. MIMO relays aim to provide improved system capacity, increases in range, and better diversity.

In this paper, we analyse the ergodic capacity of an AF MIMO two-hop system including the direct link. Most of the capacity results on two-hop MIMO relays were derived by employing asymptotic methods [5], [6], [7]. Furthermore, the random matrix results required for the MIMO relay capacity analysis are usually presented for two separate cases [8], [9] depending on whether the system is defined by a Wishart or a Pseudo-Wishart [10] distribution. However, a unified expression for the capacity of the AF MIMO two-hop system, without the source to destination link, was derived in [11].

The work of M. R. McKay was supported by Grant RPC07/08.EG16.
Our main contribution in this paper is to derive an exact expression for the capacity of an AF MIMO two-hop system including the source to destination link as shown in Fig. 1. Our expression is unified and it can be used for arbitrary numbers of antennas at the source, relay and destination. We also present simulation results to validate our analysis and the results are used to quantify the capacity improvement due to the direct link.

\section{SYSTEM MODEL}

We use the relay network topology shown in Fig. 1. The source $(S)$, relay $(R)$, and destination $(D)$ terminals are equipped with $n_{s}, n_{r}$ and $n_{d}$ antennas respectively. During the first hop, $S$ transmits (broadcasts) to $R$ and $D$ and in the second hop $R$ transmits the amplified signal from the first hop to $D$. We let the normalized channel matrices for the source-to-relay $(S \rightarrow R)$, source-to-destination $(S \rightarrow D)$, and relay-to-destination $(R \rightarrow D)$ links be given by $\boldsymbol{H}_{1} \in \mathcal{C}^{n_{r} \times n_{s}}$, $\boldsymbol{H}_{3} \in \mathcal{C}^{n_{d} \times n_{s}}$, and $\boldsymbol{H}_{2} \in \mathcal{C}^{n_{d} \times n_{r}}$, respectively. We assume that $S$ and $R$ have no channel state information and that $D$ has perfect knowledge of all channels. In addition, all channels are assumed to exhibit independent and identicallydistributed (i.i.d.) flat Rayleigh fading, and as such, the entries of the corresponding channel matrices are modeled as i.i.d. zero mean circularly symmetric complex Gaussian (ZMCSCG) random variables with unit variance. Furthermore, we assume that $R$ assists in the communication with $D$ using AF relaying. Hence, $R$ amplifies the received observation corresponding to the signal from $S$ by a factor, $b$, and retransmits it to $D$. The received signal at the destination after the two hops is then given by

$$
\boldsymbol{y}=\left[\begin{array}{c}
\sqrt{P_{3}} \boldsymbol{H}_{3} \\
\sqrt{P_{2}} \sqrt{P_{1}} b \boldsymbol{H}_{2} \boldsymbol{H}_{1}
\end{array}\right] \boldsymbol{x}+\left[\begin{array}{c}
\boldsymbol{n}_{3} \\
\sqrt{P_{2}} b \boldsymbol{H}_{2} \boldsymbol{n}_{1}+\boldsymbol{n}_{2}
\end{array}\right] .
$$

In (1), the parameters $P_{1}, P_{2}$ and $P_{3}$ are the average powers of the $S \rightarrow R, R \rightarrow D$ and $S \rightarrow D$ links, respectively, taking into account the different path loss and shadowing effects over the links. The variables $\boldsymbol{n}_{1}, \boldsymbol{n}_{2}$ and $\boldsymbol{n}_{3}$ are the noise vectors at $R$, $D$ (second-hop) and $D$ (first-hop) respectively, and $\boldsymbol{x}$ is the vector of transmit symbols. The transmit symbols are assumed i.i.d. with $E\left\{\boldsymbol{x} \boldsymbol{x}^{\dagger}\right\}=\rho \boldsymbol{I}_{n_{s}}$. The noise at $R$ and $D$ is modeled as ZMCSCG with $E\left\{\boldsymbol{n}_{1} \boldsymbol{n}_{1}^{\dagger}\right\}=\sigma_{1}^{2} \boldsymbol{I}_{n_{r}}, E\left\{\boldsymbol{n}_{2} \boldsymbol{n}_{2}^{\dagger}\right\}=\sigma_{2}^{2} \boldsymbol{I}_{n_{d}}$, 


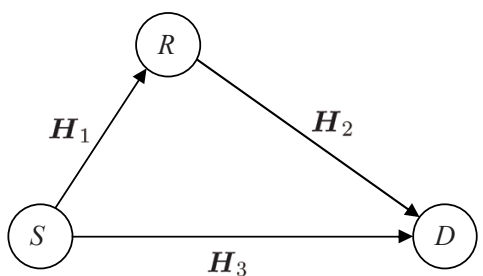

Fig. 1. MIMO relay network topology.

and $E\left\{\boldsymbol{n}_{3} \boldsymbol{n}_{3}^{\dagger}\right\}=\sigma_{3}^{2} \boldsymbol{I}_{n_{d}}$. With this information, and defining $F_{1}=\sqrt{P_{2}} \sqrt{P_{1}} b, F_{2}=\sqrt{P_{2}} b, F_{3}=\sqrt{P_{3}}$, the received signal at the destination can also be written as

$$
\boldsymbol{y}=\boldsymbol{H} \boldsymbol{x}+\boldsymbol{B} \boldsymbol{v}
$$

where

$$
\begin{gathered}
\boldsymbol{H}=\left[\begin{array}{c}
F_{3} \boldsymbol{H}_{3} \\
F_{1} \boldsymbol{H}_{2} \boldsymbol{H}_{1}
\end{array}\right], \\
\boldsymbol{B}=\left[\begin{array}{cc}
\sigma_{3}^{2} \boldsymbol{I}_{n_{d}} & \mathbf{0} \\
\mathbf{0} & \sigma_{1}^{2} F_{2}^{2} \boldsymbol{H}_{2} \boldsymbol{H}_{2}^{\dagger}+\sigma_{2}^{2} \boldsymbol{I}_{n_{d}}
\end{array}\right]^{1 / 2},
\end{gathered}
$$

and $v$ is a normalized zero mean Gaussian noise vector, which has $\boldsymbol{I}_{2 n_{d}}$ as covariance matrix.

\section{CAPACITY ANALYSIS}

The ergodic capacity of the system is given by [3] as below, (the factor $1 / 2$ accounts for the fact that information is conveyed to the destination terminal over two time slots [1])

$$
C=\frac{1}{2} E\left\{\log _{2}\left|\boldsymbol{I}_{2 n_{d}}+\rho \boldsymbol{H} \boldsymbol{H}^{\dagger}\left(\boldsymbol{B} \boldsymbol{B}^{\dagger}\right)^{-1}\right|\right\} .
$$

The singular value decomposition of $\boldsymbol{H}_{2}$ can be defined as $\boldsymbol{H}_{2}=\boldsymbol{U} \boldsymbol{D} \boldsymbol{V}^{\dagger}$, where $\boldsymbol{D}$ is an $n_{d} \times n_{r}$ diagonal matrix with $\left\{\sqrt{\nu_{1}}, \ldots, \sqrt{\nu_{l}}\right\}$ as the main diagonal elements in decreasing order and where $l=\min \left(n_{d}, n_{r}\right)$. Then, using the identity $\operatorname{det}(\boldsymbol{I}+\boldsymbol{A} \boldsymbol{B})=\operatorname{det}(\boldsymbol{I}+\boldsymbol{B} \boldsymbol{A})$ and substituting $\boldsymbol{H}_{2}=\boldsymbol{U} \boldsymbol{D} \boldsymbol{V}^{\dagger}$ into (5), the ergodic capacity can also be written as

$$
C=\frac{1}{2} E\left\{\log _{2}\left|\boldsymbol{I}_{n_{s}}+\rho \boldsymbol{U}_{t}^{\dagger} \boldsymbol{A} \boldsymbol{U}_{t}\right|\right\}
$$

where $\boldsymbol{A}=\left(\begin{array}{cc}\sigma_{3}^{-2} F_{3}^{2} \boldsymbol{I}_{n_{d}} & \mathbf{0} \\ \mathbf{0} & \boldsymbol{\Omega}\end{array}\right)$ and $\boldsymbol{U}_{t}=\left(\begin{array}{c}\boldsymbol{U}^{\dagger} \boldsymbol{H}_{3} \\ \boldsymbol{V}^{\dagger} \boldsymbol{H}_{1}\end{array}\right)$, with $\boldsymbol{\Omega}=F_{1}^{2} \boldsymbol{D}^{\dagger}\left(\sigma_{1}^{2} F_{2}^{2} \boldsymbol{D} \boldsymbol{D}^{\dagger}+\sigma_{2}^{2} \boldsymbol{I}\right)^{-1} \boldsymbol{D}$. Note that $\boldsymbol{U}_{t}$ contains i.i.d ZMCSCG entries since the unitary matrices $\boldsymbol{U}^{\dagger}$ and $\boldsymbol{V}^{\dagger}$ do not change the statistics of $\boldsymbol{H}_{3}$ and $\boldsymbol{H}_{1}$. After defining $c=$ $\sigma_{3}^{-2} F_{3}^{2}, c_{1}=\left(F_{1}^{2}-\sigma_{3}^{-2} F_{3}^{2} \sigma_{1}^{2} F_{2}^{2}\right), c_{2}=\sigma_{3}^{-2} F_{3}^{2} \sigma_{2}^{2}, c_{3}=F_{1}^{2}$, $c_{4}=\sigma_{1}^{2} F_{2}^{2}$, and $c_{5}=\sigma_{2}^{2}, \boldsymbol{\Omega}$ can be given as

$$
\boldsymbol{\Omega}= \begin{cases}\operatorname{diag}\left\{\frac{c_{3} \nu_{1}}{c_{4} \nu_{1}+c_{5}}, \ldots, \frac{c_{3} \nu_{l}}{c_{4} \nu_{l}+c_{5}}\right\} & n_{r} \leqslant n_{d} \\ \operatorname{diag}\{\frac{c_{3} \nu_{1}}{c_{4} \nu_{1}+c_{5}}, \ldots, \frac{c_{3} \nu_{l}}{c_{4} \nu_{l}+c_{5}}, \underbrace{0, \ldots, 0}_{n_{r}-n_{d}}\} & n_{r}>n_{d}\end{cases}
$$

Further, by defining $m=\max \left(n_{d}, n_{r}\right), q=n_{d}+l$ and $s=$ $\min \left(n_{s}, q\right)$, the ergodic capacity can also be expressed as

$$
C=\frac{1}{2} E\left\{\log _{2}\left|\boldsymbol{I}_{n_{s}}+\rho \widetilde{\boldsymbol{U}}_{t}^{\dagger} \widetilde{\boldsymbol{A}} \widetilde{\boldsymbol{U}}_{t}\right|\right\}
$$

where $\widetilde{\boldsymbol{U}}_{t} \in \mathcal{C}^{n_{s} \times q}$ has i.i.d ZMCSCG entries with unit variance and

$$
\widetilde{\boldsymbol{A}}=\left(\begin{array}{cc}
c \boldsymbol{I}_{n_{d}} & \mathbf{0} \\
\mathbf{0} & \operatorname{diag}\left\{\frac{c_{3} \nu_{1}}{c_{4} \nu_{1}+c_{5}}, \ldots, \frac{c_{3} \nu_{l}}{c_{4} \nu_{l}+c_{5}}\right\}
\end{array}\right) .
$$

Note that $\widetilde{\boldsymbol{A}}$ and $\widetilde{\boldsymbol{U}}_{t}$ are re-sized versions of $\boldsymbol{A}$ and $\boldsymbol{U}_{t}$ according to $n_{r} \leqslant n_{d}$ or $n_{r}>n_{d}$. Now the ergodic capacity can be written as

$$
C=\frac{s}{2 \ln (2)} \int_{0}^{\infty} \ln (1+\rho \lambda) f(\lambda) d \lambda,
$$

where $\lambda$ denotes an arbitrary eigenvalue of $\widetilde{\boldsymbol{U}}_{t}^{\dagger} \widetilde{\boldsymbol{A}} \widetilde{\boldsymbol{U}}_{t}$ and $f(\lambda)$ is the probability density function (p.d.f.) of $\lambda$. Hence, to find the ergodic capacity of the system, we need to find the arbitrary eigenvalue density, $f(\lambda)$, of the random matrix $\widetilde{\boldsymbol{U}}_{t}^{\dagger} \widetilde{\boldsymbol{A}} \widetilde{\boldsymbol{U}}_{t}$. The derivation of $f(\lambda)$ is given below.

If we assume the random diagonal matrix $\widetilde{\boldsymbol{A}}$ has all distinct eigenvalues $\boldsymbol{\mu}=\left\{\mu_{1}, \ldots, \mu_{q}\right\}$, then the conditional unordered eigenvalue p.d.f. $f(\lambda \mid \boldsymbol{\mu})$ for arbitrary numbers of antennas at the source, relay and destination can be obtained from [11] as

$$
f(\lambda \mid \boldsymbol{\mu})=\frac{1}{s \prod_{k<p}^{q}\left(\mu_{p}-\mu_{k}\right)} \sum_{k=q-s+1}^{q} \frac{\lambda^{n_{s}-q+k-1}}{\Gamma\left(n_{s}-q+k\right)}|\boldsymbol{G}|,
$$

where $G$ is a $q \times q$ matrix with entries

$$
\boldsymbol{G}_{i, j}=\left\{\begin{array}{ll}
\mu_{j}^{i-1} & i \neq k \\
\mu_{j}^{q-n_{s}-1} e^{-\frac{\lambda}{\mu_{j}}} & i=k
\end{array} .\right.
$$

However, the eigenvalues of $\widetilde{\boldsymbol{A}}$ are not all distinct but can be given as $\left\{c, \ldots, c, \mu_{1}, \ldots, \mu_{l}\right\}$, where $c$ is a constant which has multiplicity $n_{d}$, and $\mu_{k}=\frac{c_{3} \nu_{k}}{c_{4} \nu_{k}+c_{5}}$ are random variables which are unequal with probability 1 . When $\tilde{\boldsymbol{A}}$ does not have all distinct values, the conditional p.d.f. $f(\lambda \mid \boldsymbol{\mu})$ can be obtained by using the following identities on multiple derivatives,

1) If $y=x^{n}$, then the $k^{\text {th }}$ derivative of $y, y^{(k)}=(n-k+$ $1)_{k} x^{n-k}$, where $(n)_{k}$ is the Pochhammer symbol.

2) If $y=x^{n} e^{-s / x}$, then the $k^{\text {th }}$ derivative of $y, y^{(k)}=$ $e^{-s / x} \sum_{i=0}^{k} \frac{k !}{i !(k-i) !}(n-k+1)_{k-i} s^{i} x^{n-k-i}$.

These derivatives are then used to derive a modified version of (11) using the method given in [12]. With this approach $f(\lambda \mid \boldsymbol{\mu})$ can be calculated as

$$
\begin{aligned}
f(\lambda \mid \boldsymbol{\mu})= & \frac{1}{s \prod_{k<p}^{l}\left(\mu_{k}-\mu_{p}\right) \prod_{k=1}^{l}\left(c-\mu_{k}\right)^{n_{d}} \prod_{k=1}^{n_{d}-1} k !} \\
& \cdot \frac{1}{(-1)^{q(q-1) / 2}} \sum_{k=q-s+1}^{q} \frac{\lambda^{n_{s}-q+k-1}}{\Gamma\left(n_{s}-q+k\right)}|\boldsymbol{G}|,
\end{aligned}
$$

where $\Gamma_{m}(n)=\prod_{i=1}^{m} \Gamma(n-i+1)$ and $\boldsymbol{G}$ is a $q \times q$ matrix with entries given in (14). Then, using the results in (13)-(14) 


$$
\boldsymbol{G}_{i, j}=\left\{\begin{array}{ll}
\left(i-n_{d}+j\right)_{n_{d}-j} c^{i-n_{d}+j-1} & i \neq k, j=1, \ldots, n_{d} \\
\sum_{t=0}^{n_{d}-j} e^{-\frac{\lambda}{c}} \frac{\left(n_{d}-j\right) !}{t !\left(n_{d}-j-t\right) !}\left(q-n_{s}-n_{d}+j\right) n_{d}-j-t \lambda^{t} c^{q-n_{s}-n_{d}+j-1-t} & i=k, j=1, \ldots, n_{d} \\
\mu_{j-n_{d}}^{i-1} & i \neq k, j=n_{d}+1, \ldots, q \\
\mu_{j-n_{d}}^{q-n_{s}-1} e^{-\frac{\lambda}{\mu_{j-n_{d}}}} & i=k, j=n_{d}+1, \ldots, q
\end{array} .\right.
$$

we can derive the arbitrary eigenvalue p.d.f., $f(\lambda)$, as given below. Here we focus on $P_{1} \neq P_{3},\left(c_{1} \neq 0\right)$ which is of more physical interest. The special case, $P_{1}=P_{3},\left(c_{1}=0\right)$ has to be considered separately and yields a simpler result (see Appendix B).

Theorem 1: The p.d.f of an arbitrary eigenvalue $\lambda$ of $\widetilde{\boldsymbol{U}}_{t}^{\dagger} \widetilde{\boldsymbol{A}} \widetilde{\boldsymbol{U}}_{t}$ is given by

$f(\lambda)=C_{1} \sum_{i=q-s+1}^{q} \sum_{j=1}^{q}(-1)^{i+j} \frac{\lambda^{n_{s}-q+i-1}}{\Gamma\left(n_{s}-q+i\right)}\left|\boldsymbol{K}_{i, j}\right| A_{\lambda}(i, j)$,

where

$$
C_{1}=\frac{\pi^{l(l-1)}}{\mathcal{C} \Gamma_{l}(l) \mathcal{C} \Gamma_{l}(m)} \frac{(-1)^{-n_{d} l}(-1)^{l(l-1) / 2}}{s \prod_{k=1}^{n_{d}-1} k !(-1)^{q(q-1) / 2}\left(c_{3} c_{5}\right)^{l(l-1) / 2}},
$$

$\mathcal{C} \Gamma_{l}(m)$ denotes the complex multivariate gamma function, $\mathcal{C} \Gamma_{l}(m)=\pi^{l(l-1) / 2} \prod_{k=1}^{l} \Gamma(m-k+1)$, and $\boldsymbol{K}_{i, j}$ denotes the $(i, j)^{\text {th }}$ minor of $\boldsymbol{K}$ with elements given in (19). Also, $A_{\lambda}(i, j)$ is given in (17), where $\xi(x)$ in (17) is defined by

$$
\xi(x)=x^{m-l} e^{-x} \frac{\left(c_{4} x+c_{5}\right)^{q-1}}{\left(c_{1} x-c_{2}\right)^{n_{d}}} .
$$

Proof: See Appendix A.

In (19), if $c_{1}>0$, i.e. $P_{1}>P_{3}$, then $I_{A 1}=$ $\int_{-c_{2}}^{\infty} y^{v+w-n_{d}} e^{-\frac{y}{c_{1}}} d y$. Since $c_{2}>0$ the integral includes the point $y=0$ where a singularity occurs when $v+w-n_{d}<0$. In this case the individual integral diverges but the sum of integrals implicit in (15) must remain finite. Hence we compute the integral as

$$
I_{A 1}=\underbrace{\int_{-c_{2}}^{-\epsilon} y^{v+w-n_{d}} e^{-\frac{y}{c_{1}}} d y}_{I_{A 11}}+\underbrace{\int_{\epsilon}^{\infty} y^{v+w-n_{d}} e^{-\frac{y}{c_{1}} d y}}_{I_{A 12}} .
$$

where $\epsilon$ is a very small positive number close to zero. With this approach the divergent integrals cancel out in (15) and the resulting computations prove to be robust and stable. In (20) the two integrals can be evaluated as given in (21) and (22). In (21) and (22), $\operatorname{Ei}(x)$ is the exponential integral. Note also that when $c_{1}>0$, the integral in (17) has a singular point at $x=c_{2} / c_{1}$. That integral also has to be approximated as in (20), and to be consistent, the region of integration has to be $\left(0, c_{2} / c_{1}-\epsilon / c_{1}\right)$ and $\left(c_{2} / c_{1}+\epsilon / c_{1}, \infty\right)$.

If $c_{1}<0$, i.e. $P_{1}<P_{3}$, then $I_{A 1}=$ $\int_{c_{2}}^{\infty}-(-y)^{v+w-n_{d}} e^{\frac{y}{c_{1}}} d y$ in (19) and is given by

$$
I_{A 1}=\left(c_{1}\right)^{v+w-n_{d}+1} \Gamma\left(v+w-n_{d}+1,-c_{2} / c_{1}\right),
$$

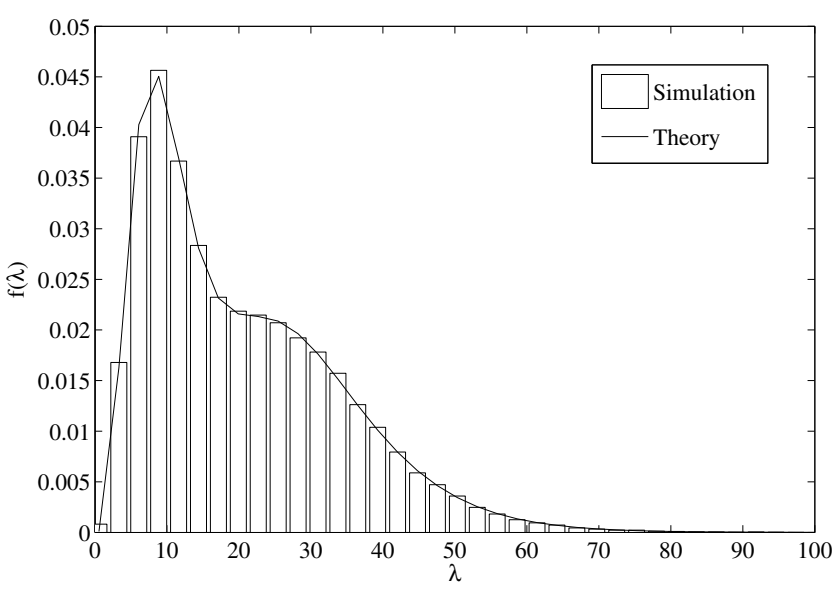

Fig. 2. Analytical and simulated p.d.f.s of the arbitrary eigenvalue of $\widetilde{\boldsymbol{U}}_{t}^{\dagger} \tilde{\boldsymbol{A}} \tilde{\boldsymbol{U}}_{t}$, with system parameters: $(3,2,3), P_{1}=P_{2}=10 \mathrm{~dB}$, and $P_{3}=5 \mathrm{~dB}$.

where $\Gamma(a, z)=\int_{z}^{\infty} e^{-x} x^{a-1} d x$ is the complementary incomplete gamma function.

Then, using the above result and (10), the ergodic capacity can be calculated as

$$
\begin{gathered}
C=\sum_{i=q-s+1}^{q} \sum_{j=1}^{q} \frac{s}{2 \ln (2)} C_{1}(-1)^{i+j}\left|\boldsymbol{K}_{i, j}\right| \\
\cdot \underbrace{\int_{0}^{\infty} \ln (1+\rho \lambda) \frac{\lambda^{n_{s}-q+i-1}}{\Gamma\left(n_{s}-q+i\right)} A_{\lambda}(i, j) d \lambda}_{I_{B}}
\end{gathered}
$$

where $I_{B}$ is given in (25). A closed form expression for the integral, $I_{C}$, in (25) is difficult to find but it can be evaluated numerically. Again, note that when $c_{1}>0$, the integral, $I_{C}$, has a singular point at $x=c_{2} / c_{1}$. We use the same approximation as in (20), and the region of integration is $\left(0, c_{2} / c_{1}-\epsilon / c_{1}\right)$ to $\left(c_{2} / c_{1}+\epsilon / c_{1}, \infty\right)$.

By using the result in (24), the ergodic capacity of the system without the direct link can also be obtained as a special case. The derivation is omitted due to space limitation.

\section{RESULTS}

The results produced in this paper are validated by using Monte Carlo simulation. In all results given, we have used the following conditions:

- The total transmitted power from the source is equal to one, i.e. $\rho=1 / n_{s}$,

- on average, the total transmitted power from the relay is equal to one, $b=\sqrt{1 /\left(n_{r} P_{1}+n_{r} \sigma_{1}^{2}\right)}$. 


$$
\begin{aligned}
& A_{\lambda}(i, j)= \begin{cases}\sum_{t=0}^{n_{d}-j} e^{-\frac{\lambda}{c}} \frac{\left(n_{d}-j\right) !}{t !\left(n_{d}-j-t\right) !}\left(q-n_{s}-n_{d}+j\right) n_{d}-j-t \lambda^{t} c^{q-n_{s}-n_{d}+j-1-t} & i=1, \ldots, q, j=1, \ldots, n_{d} \\
\int_{0}^{\infty} x^{j-n_{d}-1}\left(\frac{c_{3} x}{c_{4} x+c_{5}}\right)^{q-n_{s}-1} e^{-\frac{\lambda}{\left(\frac{c_{3} x}{c_{4} x+c_{5}}\right)} \xi(x) d x} & i=1, \ldots, q, j=n_{d}+1, \ldots, q\end{cases} \\
& \boldsymbol{K}_{i, j}=\left\{\begin{array}{ll}
\left(i-n_{d}+j\right)_{n_{d}-j} c^{i-n_{d}+j-1} & i=1, \ldots, q, j=1, \ldots, n_{d} \\
\int_{0}^{\infty} x^{j-n_{d}-1}\left(\frac{c_{3} x}{c_{4} x+c_{5}}\right)^{i-1} \xi(x) d x \triangleq I_{A}(i, j) & i=1, \ldots, q, j=n_{d}+1, \ldots, q
\end{array},\right. \\
& I_{A 12}= \begin{cases}-e^{\frac{-\epsilon}{c_{1}}} \sum_{k=0}^{v+w-n_{d}} \frac{(-1)^{v+w-n_{d}-k}\left(v+w-n_{d}\right) !(\epsilon)^{k}}{k !\left(-1 / c_{1}\right)^{v+w-n_{d}-k+1}} & v+w-n_{d} \geq 0 \\
\frac{(-1)^{n_{d}-w-v}\left(1 / c_{1}\right)^{n_{d}-w-v-1} \operatorname{Ei}\left(-\epsilon / c_{1}\right)}{\left(n_{d}-w-v-1\right) !}+\frac{e^{-\epsilon / c_{1}}}{(\epsilon)^{n d^{-w-v-1}} \sum_{k=0}^{n_{d}-w-v-2}} & v+w-n_{d}<0\end{cases}
\end{aligned}
$$

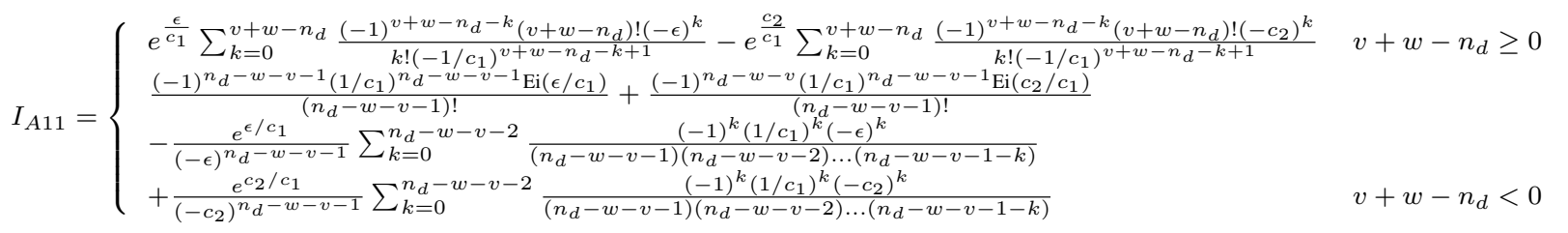

$$
\begin{aligned}
& I_{B}=\left\{\begin{array}{l}
\sum_{t=0}^{n_{d}-j} \frac{\left(n_{d}-j\right) !}{t !\left(n_{d}-j-t\right) !} \frac{\left(q-n_{s}-n_{d}+j\right)_{n}-j-t}{\Gamma\left(n_{s}-q+i\right)} c^{q-n_{s}-n_{d}+j-1-t} \\
\quad(1 / \rho)^{n_{s}+t-q+i}\left(n_{s}+t-q+i-1\right) ! e^{\frac{1}{\rho c}} \sum_{r=1}^{n_{s}+t-q+i} \Gamma\left(-\left(n_{s}+t-q+i\right)+r, 1 /(\rho c)\right)(\rho c)^{r} \quad \begin{array}{l}
i=1, \ldots, q, j=1, \ldots, n_{d} \\
i=1, \ldots, q, j=n_{d}+1, \ldots, q
\end{array} \\
I_{C} \quad
\end{array}\right.
\end{aligned}
$$

where $I_{C}=\int_{0}^{\infty} \frac{x^{j-n_{d}-1}\left(n_{s}-q+i-1\right) !}{\Gamma\left(n_{s}-q+i\right)}\left(\frac{c_{3} x}{c_{4} x+c_{5}}\right)^{q-n_{s}-1}(1 / \rho)^{n_{s}-q+i} e^{\frac{c_{4} x+c_{5}}{\rho c_{3} x}} \sum_{k=1}^{n_{s}-q+i} \frac{\Gamma\left(-\left(n_{s}-q+i\right)+k,\left(c_{4} x+c_{5}\right) /\left(\rho c_{3} x\right)\right)}{\left(\left(c_{4} x+c_{5}\right) /\left(\rho c_{3} x\right)\right)^{k}} \xi(x) d x$

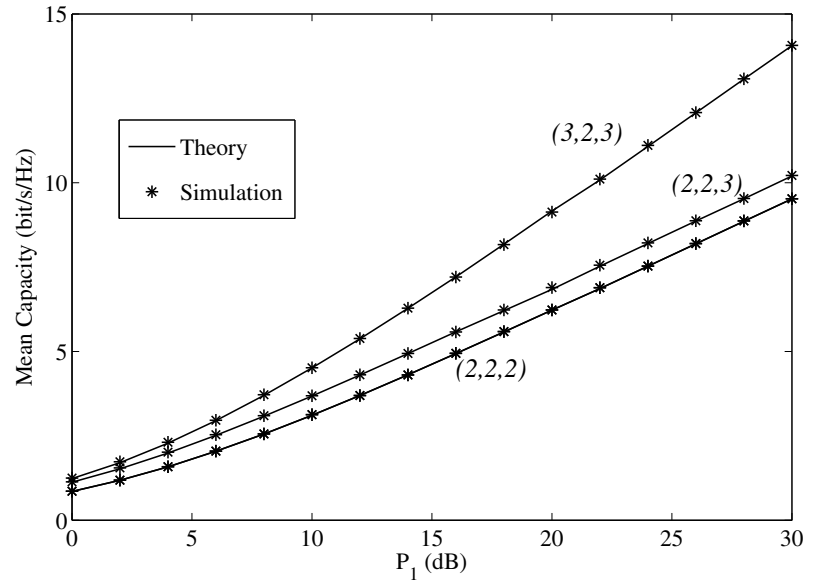

Fig. 3. Analytical and simulated ergodic capacity of the system with parameters: $P_{1}=P_{2}=1.5 P_{3}$.

Furthermore, we set $\sigma_{1}^{2}=\sigma_{2}^{2}=\sigma_{3}^{2}=1$, implying that the signal-to-noise ratios (SNR) of the links $(S \rightarrow D),(S \rightarrow R)$ and $(R \rightarrow D)$ are $P_{3}, P_{1}$ and $P_{2}$, respectively. In the results, the number of antennas used in the system is represented by the 3 -tuple $\left(n_{s}, n_{r}, n_{d}\right)$. First, in Fig. 2, we validate the result in Theorem 1 via simulation. The plots show the p.d.f. of

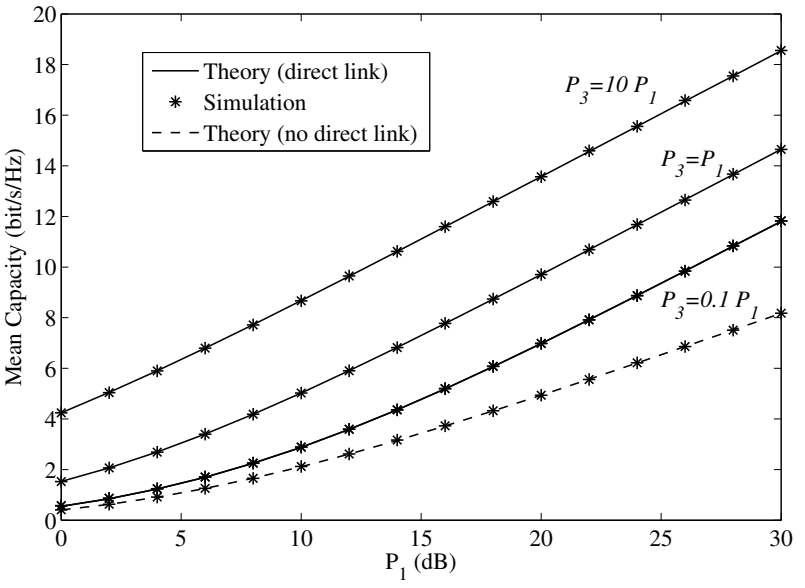

Fig. 4. Analytical and simulated ergodic capacity of the system with parameters: $(3,2,3)$ and $P_{1}=P_{2}$.

the arbitrary eigenvalue $\lambda$ with system configuration $(3,2,3)$. Figure 2 shows that the analytical results are in agreement with the simulations.

Figure 3 gives the analytical and simulated ergodic capacity of the AF MIMO system given in Fig. 1. The analytical results are based on (24) and the results are given for the system 


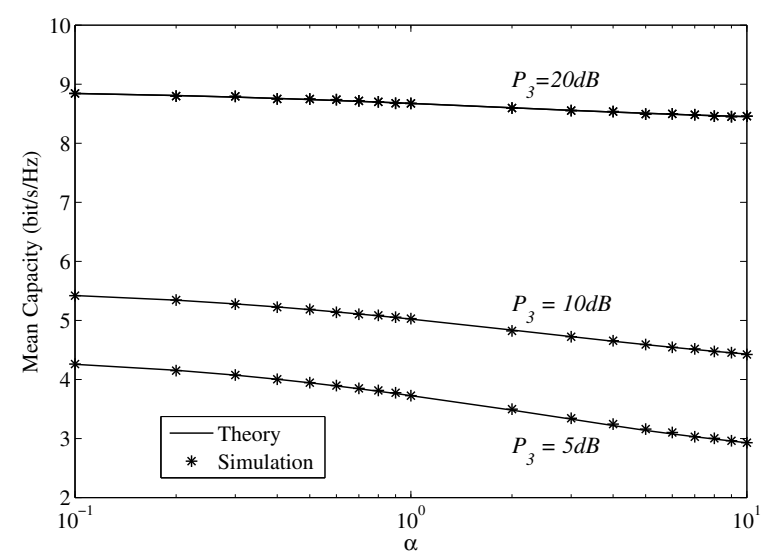

Fig. 5. Analytical and simulated ergodic capacity of a $(3,2,3)$ system vs. $\alpha$, where $P_{1}=\alpha P_{2}=10 \mathrm{~dB}$. Also shown is the $S \rightarrow D$ link power, $P_{3}$.

configurations: $(2,2,2),(2,2,3)$ and $(3,2,3)$. The results are given as a function of the SNR in the links as $P_{1}=P_{2}=$ $1.5 P_{3}$. We see that the analytical results exactly match the simulations.

Figure 4 gives the performance of the analytical and simulated ergodic capacity of the system with configuration $(3,2,3)$, when the $S \rightarrow D$ link strength varies. The results show that the capacity of the system improves with increases in the $S \rightarrow D$ signal strength due to diversity improvement. Note that when $P_{3}=P_{1}$, the results in Appendix B are used to generate analytical results. We have also included the performance of the system without the direct link. Hence, Fig. 4 shows the performance gains of the system due to the inclusion of the direct link. Again the analytical results exactly match with the simulations.

Finally, Fig. 5 shows the performance of a $(3,2,3)$ system with varying $R \rightarrow D$ link SNR. The SNR have the relationship $P_{1}=\alpha P_{2}=10 \mathrm{~dB}$. The results show that when $P_{3}$ (the SNR of the $S \rightarrow D$ link) is high there is not much improvement in capacity even though $P_{2}$ is increased. Also when $P_{3}$ is weak the capacity improvement due to increases in $P_{2}$ is more obvious.

\section{CONCLUSIONS}

The paper presents an ergodic capacity analysis of an $\mathrm{AF}$ MIMO two-hop system including the source to destination link. We first derived an expression for the probability density function of the unordered eigenvalue of the system and from that, a closed form expression for the ergodic capacity of the system is derived. We also validated the analysis by using simulations and both results match exactly. The results also show that having the direct link improves the capacity due to diversity and quantifies this improvement.

\section{APPENDIX}

\section{A. Proof of Theorem 1}

The p.d.f. of $\lambda, f(\lambda)$, can be calculated by using the result in (13). The eigenvalues of $\widetilde{\boldsymbol{A}}, \boldsymbol{\mu}=\left\{c, \ldots, c, \mu_{1}, \ldots, \mu_{l}\right\}$, are related to the eigenvalues of $\boldsymbol{H}_{2} \boldsymbol{H}_{2}^{\dagger}, \boldsymbol{\nu}=\left\{\nu_{1}, \ldots, \nu_{l}\right\}$ via, $\mu_{k}=\frac{c_{3} \nu_{k}}{c_{4} \nu_{k}+c_{5}}$. Then, using the result for $f(\lambda \mid \boldsymbol{\mu})$ in (13), $f(\lambda \mid \nu)$ can be obtained by substituting $\mu_{k}=\frac{c_{3} \nu_{k}}{c_{4} \nu_{k}+c_{5}}$ in $f(\lambda \mid \boldsymbol{\mu})$, as fixing the values of $\boldsymbol{\mu}$ is equivalent to fixing the values of $\boldsymbol{\nu}$. Then using $f(\lambda \mid \boldsymbol{\nu})$ and $f(\boldsymbol{\nu})$, the eigenvalue p.d.f $f(\lambda)$ can be derived as shown below.

The matrix $\boldsymbol{H}_{2} \boldsymbol{H}_{2}^{\dagger}$ is Wishart or pseudo-Wishart [10] depending on the dimension, $n_{d} \times n_{r}$, of $\boldsymbol{H}_{2}$. However, the non-zero eigenvalues of $\boldsymbol{H}_{2} \boldsymbol{H}_{2}^{\dagger}$ are the same irrespective of whether the matrix is Wishart or pseudo-Wishart. Hence the non-zero unordered eigenvalue p.d.f of $\boldsymbol{H}_{2} \boldsymbol{H}_{2}^{\dagger}$ can be given as [13]

$$
f(\boldsymbol{\nu})=\frac{\pi^{l(l-1)}}{l ! \mathcal{C} \Gamma_{l}(l) \mathcal{C} \Gamma_{l}(m)} \prod_{k=1}^{l} \nu_{k}^{m-l} e^{-\nu_{k}} \prod_{k<p}^{l}\left(\nu_{k}-\nu_{p}\right)^{2} .
$$

Using the result in (13), the conditional p.d.f. $f(\lambda \mid \boldsymbol{\nu})$ can be obtained by substituting $\mu_{k}=\frac{c_{3} \nu_{k}}{c_{4} \nu_{k}+c_{5}}$ in (13) as

$$
\begin{aligned}
f(\lambda \mid \boldsymbol{\nu}) & =\frac{1}{s \prod_{k=1}^{n_{d}-1} k !(-1)^{q(q-1) / 2}\left(c_{3} c_{5}\right)^{l(l-1) / 2}} \\
& \times \frac{1}{\prod_{k<p}^{l}\left(\nu_{k}-\nu_{p}\right) \prod_{k=1}^{l} \frac{\left(\left(c c_{4}-c_{3}\right) \nu_{k}+c c_{5}\right)^{n} d}{\left(c_{4} \nu_{k}+c_{5}\right)^{n_{d}+l-1}}} \\
& \times \sum_{k=q-s+1}^{q} \frac{\lambda^{n_{s}-q+k-1}}{\Gamma\left(n_{s}-q+k\right)}|\boldsymbol{G}| .
\end{aligned}
$$

Then, using the relation $f(\lambda, \boldsymbol{\nu})=f(\boldsymbol{\nu}) f(\lambda \mid \boldsymbol{\nu}), f(\lambda, \boldsymbol{\nu})$ can be given as in (28). In (28), $\prod_{k<p}^{l}\left(\nu_{k}-\nu_{p}\right)=$ $(-1)^{l(l-1) / 2}\left|\Phi_{j}\left(\nu_{i}\right)\right|, \Phi_{i}\left(\nu_{j}\right)=\nu_{j}^{i-1}$,

$$
C_{0}=\frac{\pi^{l(l-1)}}{l ! \mathcal{C} \Gamma_{l}(l) \mathcal{C} \Gamma_{l}(m)} \frac{(-1)^{-n_{d} l}(-1)^{l(l-1) / 2}}{s \prod_{k=1}^{n_{d}-1} k !(-1)^{q(q-1) / 2}\left(c_{3} c_{5}\right)^{l(l-1) / 2}},
$$

$$
\xi\left(\nu_{k}\right)=\nu_{k}^{m-l} e^{-\nu_{k}} \frac{\left(c_{4} \nu_{k}+c_{5}\right)^{q-1}}{\left(c_{1} \nu_{k}-c c_{5}\right)^{n_{d}}}
$$

and $\boldsymbol{G}$ is a $q \times q$ matrix with entries given in (31).

Now $f(\lambda)$ can be obtained by integrating over all $\nu_{j}$ by using the method described in Lemma 2 of [14] as,

$$
\begin{aligned}
f(\lambda) & =C_{0} \sum_{k=q-s+1}^{q} \frac{\lambda^{n_{s}-q+k-1}}{\Gamma\left(n_{s}-q+k\right)} \\
& \times \int_{0}^{\infty} \ldots \int_{0}^{\infty} \prod_{k=1}^{l} \xi\left(\nu_{k}\right)\left|\Phi_{i}\left(\nu_{j}\right)\right||\boldsymbol{G}| d \nu_{1} \ldots d \nu_{l} \\
& \triangleq \underbrace{C_{0} l !}_{C_{1}} \sum_{k=q-s+1}^{q} \frac{\lambda^{n_{s}-q+k-1}}{\Gamma\left(n_{s}-q+k\right)}|\boldsymbol{\Psi}|
\end{aligned}
$$

where $\Psi$ is a $q \times q$ matrix with entries given in (33). $I_{A}(i, j)$ in (33) is given in (19). Finally, we obtain the result in Theorem 1 by using the Laplace expansion of (32).

\section{B. Ergodic Capacity when $P_{1}=P_{3}$}

In this special case, when $P_{1}=P_{3}$, the ergodic capacity of the system can be obtained by using (24). However, $\boldsymbol{K}_{i, j}$ and $I_{B}$ in (24) have to be re-evaluated by substituting $c_{1}=0$. 


$$
\begin{aligned}
& f(\lambda, \boldsymbol{\nu})=\frac{\pi^{l(l-1)}}{l ! \mathcal{C} \Gamma_{l}(l) \mathcal{C} \Gamma_{l}(m)} \frac{(-1)^{-n_{d} l}}{s \prod_{k=1}^{n_{d}-1} k !(-1)^{q(q-1) / 2}\left(c_{3} c_{5}\right)^{l(l-1) / 2}} \prod_{k=1}^{l} \nu_{k}^{m-l} e^{-\nu_{k}} \frac{\left(c_{4} \nu_{k}+c_{5}\right)^{q-1}}{\left(c_{1} \nu_{k}-c c_{5}\right)^{n_{d}}} \prod_{k<p}^{l}\left(\nu_{k}-\nu_{p}\right) \sum_{k=q-s+1}^{q} \frac{\lambda^{n_{s}-q+k-1}}{\Gamma\left(n_{s}-q+k\right)}|\boldsymbol{G}| \\
& \triangleq C_{0} \sum_{k=q-s+1}^{q} \frac{\lambda^{n_{s}-q+k-1}}{\Gamma\left(n_{s}-q+k\right)} \prod_{k=1}^{l} \xi\left(\nu_{k}\right)\left|\Phi_{i}\left(\nu_{j}\right)\right||\boldsymbol{G}| \text {. } \\
& \boldsymbol{G}_{i, j}=\left\{\begin{array}{ll}
\left(i-n_{d}+j\right)_{n_{d}-j} c^{i-n_{d}+j-1} & i \neq k, j=1, \ldots, n_{d} \\
\sum_{t=0}^{n_{d}-j} e^{-\frac{\lambda}{c}} \frac{\left(n_{d}-j\right) !}{t !\left(n_{d}-j-t\right) !}\left(q-n_{s}-n_{d}+j\right)_{n_{d}-j-t} \lambda^{t} c^{q-n_{s}-n_{d}+j-1-t} & i=k, j=1, \ldots, n_{d} \\
\left(\frac{c_{3} \nu_{j-n_{d}}}{c_{4} \nu_{j-n_{d}}+c_{5}}\right)^{i-1} & i \neq k, j=n_{d}+1, \ldots, q \\
\left(\frac{c_{3} \nu_{j-n_{d}}}{c_{4} \nu_{j-n_{d}}+c_{5}}\right)^{q-n_{s}-1} e^{-\frac{\lambda\left(c_{4} \nu_{\left.j-n_{d}+c_{5}\right)}\right.}{c_{3} \nu_{j-n_{d}}}} & i=k, j=n_{d}+1, \ldots, q
\end{array} .\right. \\
& \mathbf{\Psi}_{i, j}=\left\{\begin{array}{ll}
\left(i-n_{d}+j\right)_{n_{d}-j} c^{i-n_{d}+j-1} & i \neq k, j=1, \ldots, n_{d} \\
\sum_{t=0}^{n_{d}-j} e^{-\frac{\lambda}{c}} \frac{\left(n_{d}-j\right) !}{t !\left(n_{d}-j-t\right) !}\left(q-n_{s}-n_{d}+j\right)_{n_{d}-j-t} \lambda^{t} c^{q-n_{s}-n_{d}+j-1-t} & i=k, j=1, \ldots, n_{d} \\
\int x^{j-n_{d}-1}\left(\frac{c_{3} x}{c_{4} x+c_{5}}\right)^{i-1} \xi(x) d x \triangleq I_{A}(i, j) & i \neq k, j=n_{d}+1, \ldots, q \\
\int x^{j-n_{d}-1}\left(\frac{c_{3} x}{c_{4} x+c_{5}}\right)^{q-n_{s}-1} e^{-\frac{\lambda\left(c_{4} x+c_{5}\right)}{c_{3} x}} \xi(x) d x & i=k, j=n_{d}+1, \ldots, q
\end{array} .\right.
\end{aligned}
$$

$$
\boldsymbol{K}_{i, j}= \begin{cases}\left(i-n_{d}+j\right)_{n_{d}-j} c^{i-n_{d}+j-1} & i=1, \ldots, q, j=1, \ldots, n_{d} \\ \int_{0}^{\infty} x^{j-n_{d}-1}\left(\frac{c_{3} x}{c_{4} x+c_{5}}\right)^{i-1} \xi(x) d x \triangleq I_{A}(i, j) & i=1, \ldots, q, j=n_{d}+1, \ldots, n_{d}+l\end{cases}
$$

where $I_{A}(i, j)=c_{3}^{i-1}\left(-c_{2}\right)^{-n_{d}} \sum_{v=0}^{q-i}\left(\begin{array}{c}q-i \\ v\end{array}\right)\left(c_{4}\right)^{v}\left(c_{5}\right)^{q-i-v} \Gamma(v+j+i+m-q-1)$.

$$
I_{B}=\left\{\begin{array}{ll}
\sum_{t=0}^{n_{d}-j} \frac{\left(n_{d}-j\right) !}{t !\left(n_{d}-j-t\right) !} \frac{\left(q-n_{s}-n_{d}+j\right)_{n_{d}-j-t}}{\Gamma\left(n_{s}-q+i\right)} c^{q-n_{s}-n_{d}+j-1-t}(1 / \rho)^{n_{s}+t-q+i} & \\
\left(n_{s}+t-q+i-1\right) ! e^{\frac{1}{\rho c}} \sum_{r=1}^{n_{s}+t-q+i} \Gamma\left(-\left(n_{s}+t-q+i\right)+r, 1 /(\rho c)\right)(\rho c)^{r} & i=1, \ldots, q, j=1, \ldots, n_{d} \\
I_{C} & i=1, \ldots, q, j=n_{d}+1, \ldots, q
\end{array},\right.
$$

where $I_{C}=\frac{2 c_{3}^{q-n_{s}-1}}{\left(-c_{2}\right)^{n} d} \sum_{v=0}^{n_{s}}\left(\begin{array}{c}n_{s} \\ v\end{array}\right) c_{4}^{v} c_{5}^{n_{s}-v} \int_{0}^{\infty} \ln (1+\rho \lambda) \frac{\lambda^{n_{s}-q+i-1}}{\Gamma\left(n_{s}-q+i\right)} e^{-\frac{\lambda c_{4}}{c_{3}}}\left(\frac{\lambda c_{5}}{c_{3}}\right)^{\frac{v+j+m-n_{s}-1}{2}} K_{v+j+m-n_{s}-1}\left(2 \sqrt{\frac{\lambda c_{5}}{c_{3}}}\right) d \lambda$.

Then, for this case, $\boldsymbol{K}_{i, j}$ and $I_{B}$ can be evaluated as given in (34) and (35), respectively. Then, substituting (34) and (35) in (24), the ergodic capacity of the system when $P_{1}=P_{3}$ can be obtained.

\section{REFERENCES}

[1] R. U. Nabar, H. Bölcskei, and F. Kneubuhler, "Fading relay channels: Performance limits and space-time signal design," IEEE J. Select. Areas Commun., vol. 22, no. 6, pp. 1099-1109, Aug. 2004.

[2] A. Sendonaris, E. Erkip, and B. Aazhang, "User cooperation diversity - part 1: System description," IEEE Trans. Commun., vol. 51, no. 11, pp. 1927-1938, Nov. 2003.

[3] M. Herdin, "MIMO amplify-and-forward relaying in correlated MIMO channels," in Proc. Int. Conf. on Inform. Commun. and Signal Processing, Bangkok, Thailand, Dec. 6-9, 2005, pp. 796-800.

[4] X. Tang and Y. Hua, "Optimal design of non-regenerative MIMO wireless relays," IEEE Trans. Wireless Commun., vol. 6, no. 4, pp. 13981407, April 2007.

[5] H. Bölcskei, R. U. Nabar, O. Oyman, and A. Paulraj, "Capacity scaling laws in MIMO relay networks," IEEE Trans. Wireless Commun., vol. 5, no. 6, pp. 1433-1444, June 2006.

[6] J. Wagner, B. Rankov, and A. Wittneben, "On the asymptotic capacity of the Rayleigh fading amplify-and-forward MIMO relay channel," in Proc. IEEE Int. Symp. Information Theory (ISIT), Nice, France, June 24-29, 2007, pp. 2711-2715.
[7] V. Morgenshtern and H. Bölcskei, "Crystallization in large wireless networks," IEEE Trans. Inf. Theory, vol. 53, no. 10, pp. 3319-3349, Oct. 2007.

[8] A. Maaref and S. Aïssa, "Eigenvalue distributions of Wishart-type random matrices with application to the performance analysis of MIMO MRC systems," IEEE Trans. Wireless Commun., vol. 6, no. 7, pp. 26782689, July 2007.

[9] G. Alfano, A. Tulino, A. Lozano, and S. Verdú, "Capacity of MIMO channels with one-sided correlation," in Proc. IEEE Int. Symp. on Spread Spectrum Techniques and Applications (ISSSTA), Sydney, Australia, Aug. 30-Sep. 2, 2004, pp. 515-519.

[10] R. K. Mallik, "The pseudo-Wishart distribution and its application to MIMO systems," IEEE Trans. Inf. Theory, vol. 49, no. 10, pp. 27612769, 2003.

[11] S. Jin, M. R. McKay, C. Zhong, and K.-K. Wong, "Ergodic capacity analysis of amplify-and-forward MIMO dual-hop systems," in Proc. IEEE Int. Symp. Information Theory (ISIT), Toronto, Canada, July 611, 2008, pp. 1903-1907.

[12] M. Chiani, M. Z. Win, and H. Shin, "Capacity of MIMO systems in the presence of interference," in Proc. IEEE Global Telecommunications Conf., San Francisco, California, USA, Nov. 30-Dec. 1, 2006, pp. 1-6.

[13] T. Ratnarajah, R. Vaillancourt, and M. Alvo, "Complex random matrices and Rayleigh channel capacity," Commun. Inf. Syst., vol. 3, pp. 119-138, 2003.

[14] H. Shin, M. Z. Win, J. H. Lee, and M. Chiani, "On the capacity of doubly correlated MIMO channels," IEEE Trans. Wireless Commun., vol. 5, no. 8, pp. 2253-2265, Aug. 2006. 